\title{
Syndromes cholestatiques familiaux
}

\author{
Richard Thompson \\ Paediatric Hepatology, King's College London School of Medicine, Londres, Royaume-Uni
}

\section{Mots-clés}

Syndromes cholestatiques familiaux $\cdot$ Syndrome d'Alagille $\cdot$ Syndrome d'arthrogrypose $\cdot$ Syndrome de dysfonctionnement rénal et de cholestase $\cdot$ Cholestase intra-hépatique familiale de type $1 \cdot$ Déficit en pompe d'exportation des sels biliaires - Déficit en protéine 3 de résistance multidrogue

\section{Résumé}

Le terme «familial» est malheureux. Bien que la définition implique "héréditaire», elle sous-entend que l'affection est habituellement survenue chez d'autres membres de la famille. Pour un grand nombre des affections décrites dans le présent article, il n'en est souvent pas le cas, et, pour cette raison, des affections «familiales» peuvent être ignorées lors de la démarche diagnostique. La cholestase est définie de façon différente par les cliniciens, les anatomopathologistes et les biochimistes. De plus, la définition d'une hépatopathie cholestatique varie très souvent pour les hépatologues traitant des enfants ou des adultes. Les mécanismes pathogènes sont cependant de plus en plus similaires. L'atrésie des voies biliaires, qui est l'hépatopathie cholestatique à laquelle les pédiatres sont le plus souvent confrontés, est nettement différente de la plupart des maladies car il paraît n'exister aucun équivalent à "début tardif», et car cette affection n'est quasiment jamais familiale. Plusieurs groupes coopératifs internationaux ont été constitués dans l'espoir de surmonter ces obstacles (http://www.barcnetwork.org/, http://www.orpha.net/nestasso/EFBAR/, http://www.biliaryatresia.com/index_frameset_ebar.html). Dans sa pratique quotidienne, I'hépatologue pédiatre est confronté à un groupe de nourrissons ictériques chez qui la cause n'est pas facilement apparente. Sous de nombreux aspects, cette si- tuation a été aggravée par le fait, que nous savions depuis longtemps qu'une proportion significative s'améliorera spontanément, tandis que l'évolution sera défavorable chez d'autres patients. Au cours des premiers mois de la vie, il demeure difficile de prévoir l'évolution chez un patient donné. La cause sous-jacente, au moins dans le groupe dont l'évolution est péjorative, devient apparente dans un nombre de plus en plus important de cas. La plus grande partie des améliorations des possibilités diagnostiques dans ce groupe d'enfants est la conséquence de progrès des connaissances de l'étiologie génétique. Il est également devenu manifeste que I'hépatopathie cholestatique d'origine génétique n'est pas limitée à la population pédiatrique. L'étendue précise de la contribution de facteurs génétiques à l'apparition d'une forme à «début tardif» commence seulement à être mieux connue.

Copyright $\odot 2009$ Nestec Ltd., Vevey/S. Karger AG, Basel

La génétique n'est pas encore la première étape dans la détermination d'un diagnostic chez un nourrisson cholestatique, mais pourrait l'être bientôt [4]. Les affections cholestatiques sont désormais classées en fonction de leur cause génétique, ce qui facilite l'identification de variations phénotypiques. Ces différences phénotypiques améliorent autant le diagnostic que le pronostic. Elles aident à décider du type de situation d'un enfant, ou au moins suggèrent quel gène ou protéine devrait être exploré. Le phénotype s'étend au pronostic et, particulièrement, aux résultats du traitement, et une meilleure connaissance de ces facteurs aide tout autant le clinicien que la famille. Le tableau 1 récapitule les principaux syndromes cholestatiques familiaux.

\section{KARGER \\ Fax +4161306 1234 \\ E-Mail karger@karger.ch}

www.karger.com
C 2009 Nestec Ltd., Vevey/S. Karger AG, Basel

0250-9644/08/0663-0121\$24.50/0

Accessible en ligne à:

www.karger.com/anf
Richard Thompson

Institute of Liver Studies

King's College Hospital

Denmark Hill, London SE5 9RS (UK)

E-Mail richard.j.thompson@kcl.ac.uk 
Tableau 1. Principaux syndromes cholestatiques familiaux

\begin{tabular}{|c|c|c|c|c|}
\hline \multirow[t]{2}{*}{ Syndrome d'Alagille } & \multirow[t]{2}{*}{ Dysplasie artério-hépatique } & $J A G 1$ & Jagged 1 & $\begin{array}{l}\text { Affection multiviscérale, phénotype variable, } \\
\text { mutations hétérozygotes de l'un ou l'autre gène }\end{array}$ \\
\hline & & NOTCH2 & Notch 2 & Pénétrance variable \\
\hline Syndrome ARC & Syndrome de Nezelof & $V P S 33 B$ & VPS33B & Affection multiviscérale, cholestase discrète \\
\hline Déficit en FIC1 & Maladie de Byler, CIFP1 & ATP8B1 & FIC1 & Affection multiviscérale, sévérité très variable \\
\hline
\end{tabular}

\section{Syndrome d'Alagille}

Le diagnostic de syndrome d'Alagille a toujours été clinique. Dans la plupart des cas, des études génétiques ne seraient pas nécessaires au diagnostic chez un patient donné. Des mutations ont été tout d'abord identifiées dans JAGGED1, qui code pour un ligand de la voie de signalement Notch [5, 6]. Par la suite, chez d'autres patients, des mutations ont été décelées dans le gène codant pour l'un des récepteurs Notch, NOTCH2 [7]. Dans l'ensemble, des mutations ont été uniquement décelées sur un seul allèle du gène en question. Une perte des deux allèles serait sans doute létale et des données obtenues chez la souris sont certainement en faveur de cette hypothèse [8]. Il est désormais clair qu'un diagnostic génétique peut être réalisé chez la grande majorité de ces sujets [9]. D’une façon ou d'une autre, cette démarche a seulement compliqué la situation, notamment car toutes les données publiées concernant la fréquence des différentes caractéristiques et, fait plus important, les données sur l'évolution concernent des patients chez qui le diagnostic a été posé de façon clinique. Une "cause» génétique identifiable peut être déterminée chez la plupart des patients, mais l'on sait depuis longtemps que des membres de sa famille peuvent présenter des caractéristiques similaires, bien que plus discrètes. Il est désormais clair que ces parents présentent souvent la même anomalie génétique. Il serait certainement difficile de soutenir que ces sujets sont atteints de syndrome d'Alagille. Ce problème devient maximal si l'on réalise que la fréquence des mutations des gènes impliqués est totalement inconnue dans la population générale. De ce fait, le diagnostic doit probablement demeurer clinique jusqu'à ce que l'on connaisse la vraie pénétrance des mutations et les mécanismes qui déterminent le phénotype.

\section{Syndrome d'arthrogrypose, dysfonctionnement rénal et cholestase}

Le syndrome d'arthrogrypose, dysfonctionnement rénal et cholestase (ARC) est heureusement rare. Il représente cependant un important mécanisme pathologique. Il a été décrit au plan clinique, de la même façon que le syndrome d'Alagille. Le phénotype décrit était très sévère, particulièrement en ce qui concerne l'arthrogrypose, et ce bien que l'hépatopathie ait été relativement bénigne. Le gène muté dans la majorité des cas a été identifié comme $V P S 33 B$ [10]. Les mutations sont récessives, comme dans le cas de toutes les affections décrites dans le restant du présent article. L'on sait depuis longtemps qu'un grand nombre des fonctions de l'hépatocyte sont hautement polarisées, des fonctions très différentes s'exerçant au niveau des membranes basolatérales et canaliculaires (fig. 1). La protéine pour laquelle VPS33B code paraît intervenir dans le tri correct de certaines protéines au domaine canaliculaire et n'est pas par elle-même un transporteur transmembranaire. Il semble hautement probable que d'autres phénotypes, peut-être similaires, se manifesteront si d'autres protéines de cette voie sont anormales. Cependant, comme dans le cas du syndrome d'Alagille, des patients ne présentant pas le phénotype complet ont été déjà identifiés [11]. Ce fait est important, car ces patients n’auraient pas été précédemment classés comme ARC, ne satisfaisant pas aux critères cliniques. Ici encore, cette situation soulève la question, demeurant sans réponse, du nombre de patients présentant un phénotype moins sévère et porteurs de mutations de ces gènes.

\section{Déficit en protéine FIC1}

FIC1 (Familial intrahepatic cholestasis 1, cholestase intra-hépatique familiale de type 1) est le nom généralement donné à la protéine pour laquelle $A T P 8 B 1$ code [12]. Cette 


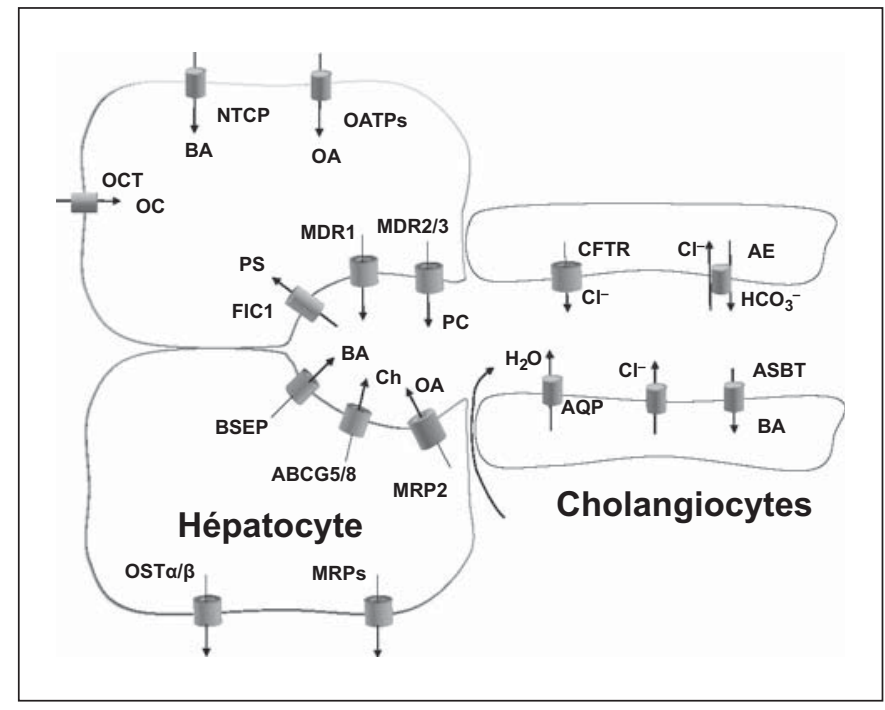

Fig. 1. Principaux transporteurs intervenant dans la formation de la bile dans les membranes des hépatocytes et des cholangiocytes, dont un grand nombre sont désormais associés à la cholestase familiale. ABCG5/8 = Transporteur hétérodimérique du cholestérol; $\mathrm{AE}=$ échangeur d'anions; $\mathrm{ASBT}=$ transporteur apical sodium-dépendent des acides biliaires; $\mathrm{AQP}=$ aquaporine; $\mathrm{BA}=$ acides biliaires; CFTR = régulateur de la conductance transmembranaire - mucoviscidose; $\mathrm{Ch}=$ cholestérol; FIC1 = protéine cholestase intrahépatique familiale de type $1 ; \mathrm{MDR}=$ protéine de résistance multidrogue; $\mathrm{MRP}=$ protéine associée à une résistance multidrogue; NTCP = polypeptide cotransporteur du sodium/ taurocholate; $\mathrm{OA}=$ anions organiques; OATP = polypeptide transporteur d'anions organiques; $\mathrm{OC}=$ cations organiques; OCT $=$ transporteur de cations organiques; OST $=$ transporteur hétérodimérique de solutés organiques; PS = phosphatidylsérine.

nomenclature génétique simple indique que la protéine devrait être une ATPase de type P et serait donc proche du gène de la maladie de Wilson. Cependant, comme nous le verrons plus loin, sa fonction est probablement très différente. Un déficit en FIC1 a été décrit comme la cause de la cholestase intra-hépatique récidivante bénigne (CIRB) et de la cholestase intra-hépatique familiale progressive (CIFP). En fait, il est évident que certains patients se situent entre ces deux définitions et que le déficit en FIC1 correspond à un spectre allant d'hépatopathies bénignes à sévères [13]. Ce point est cliniquement important, car une hépatopathie peut progresser, même quand le diagnostic initial a été celui d'une CIRB, souvent sur la base d'une présentation tardive. De ce fait, le terme «CIRB» est quelque peu trompeur et peut être faussement rassurant tant pour les patients que pour leurs soignants.

De nombreuses ATPases de type P sont des transporteurs d'ions, comme dans le cas de la protéine associée à la maladie de Wilson. D’autre part, la FIC1 paraît être l'une des aminophospholipides flippases (ou translocases). L'exigence du maintien d'une asymétrie lipidique dans les membranes biologiques est manifeste, et, de ce fait, plusieurs de ces protéines existent probablement, exprimées dans divers tissus. La répartition correcte des lipides entre les bicouches pourrait être essentielle pour le tri des protéines vers le compartiment correct. De même, il est probable qu'une composition lipidique correcte de la membrane plasmatique soit essentielle pour la fonction normale d'autres protéines à ce niveau. Un déficit en FIC1 se manifeste non seulement par une hépatopathie cholestatique, mais également par un ensemble très variable d'autres caractéristiques. Les plus communes sont une diarrhée, une perte de l'audition, un dysfonctionnement tubulaire rénal et un retard de croissance. Il est un peu surprenant de noter que des sujets peuvent présenter des manifestations sévères d'un aspect de la maladie, avec des atteintes minimales d'un autre appareil ou organe. La nature multiviscérale de la maladie est confortée par l'expression très répandue du gène ATP8B1 [12]. Dans le foie, plusieurs protéines sont absentes ou ne sont présentes qu'en quantités significativement réduites dans la membrane canaliculaire chez les patients atteints de déficit en FIC1. Ce fait est en accord avec l'hypothèse selon laquelle des anomalies des lipides membranaires aboutissent à des défaillances du processus de tri des protéines, comme dans le cas du syndrome d'ARC. Pourtant, aucune des protéines manquantes n'est connue comme indispensable pour la formation de la bile ni associée à une cholestase. En particulier, le marquage immunohistochimique de la pompe d'exportation des sels biliaires (BSEP, discutée de façon plus détaillée plus bas) paraît normal dans le déficit en FIC1. Des données récentes sont cependant en faveur d'une autre hypothèse [14]. Bien que largement basés sur un modèle murin, ces travaux suggèrent que la protéine FIC1 facilite le déplacement d'aminophospholipides du feuillet externe au feuillet interne de la membrane plasmatique [14], comme on le pensait. La membrane canaliculaire normale doit maintenir une résistance elévée aux détergents afin d'éviter des lésions dues à l'effet détergent de la bile elle-même. La présence d'aminophospholipides en excès dans le feuillet externe pourrait réduire cette résistance aux détergents. Utilisant une technique de perfusion à court terme ex vivo d'acides biliaires, ces auteurs ont observé que la disponibilité des aminophospholipides pour extraction par les acides biliaires était fortement accrue dans le modèle knockout. De plus, certaines des ectoenzymes canaliculaires des foies knockout ont été éluées d'une façon dépendante des sels biliaires. Ce sont certaines des mêmes 
enzymes dont l'absence dans le foie humain avait été précédemment montrée. Ces données permettent de penser que ces protéines ont été éliminées de la membrane canaliculaire du foie humain atteint et n'ont pas été sujettes à une anomalie d'acheminement ou de ciblage. Cette hypothèse n'a pas encore été démontrée chez l'homme et n'explique toujours pas la cholestase, car aucune formation de transporteur capital pour la bile ne s'est avérée absente. Pour ces protéines volumineuses, il semble très probable que c'est leur fonction qui est affectée par la composition anormale des lipides de la membrane canalaire. Il peut en être de même dans les autres organes atteints. Dansl'oreille, le rein ou le pancréas, ce n'est manifestement pas l'action des protéines d'élimination des acides biliaires qui aboutit au phénotype. Il semble peu douteux qu'une composition correcte des lipides de la membrane est capitale pour de nombreuses fonctions. Nous demeurons face à la difficulté supplémentaire de phénotypes hautement variables. Nous savons qu'il existe plusieurs autres transporteurs des aminophospholipides dans le protéome humain. Il semble probable que, dans le déficit en FIC1, la maladie se manifeste uniquement dans les sites où d'autres protéines ne compensent pas adéquatement la perte de la fonction FIC1. Les manifestations extra-hépatiques du déficit en FIC1 limitent l'utilité d'une transplantation hépatique. En particulier, une diarrhée peut s'aggraver, et le retard de croissance peut n'être aucunement influencé. Le prurit invalidant peut être suffisant pour justifier l'intervention, mais celle-ci ne peut être considérée comme curative.

Le déficit en FICl a été appelé «maladie de Byler», du nom de la famille Amish dans laquelle il a été décrit pour la première fois. La bile de maladie de Byler est la description des contenus canaliculaires observés en microscopie électronique de transmission dans le déficit en FIC1. Ces agrégats inhabituels ne sont pas particulièrement spécifiques de cette affection, mais l'évoquent très fortement. La souris knockout présente une morphologie indiquant une bile de maladie de Byler quand elle est alimentée en acide cholique [14]. Chez la souris, les aspects sont hautement évocateurs de la formation de composants granuleux à partir de microvillosités lésées, provenant de la membrane canaliculaire. Cette perte de surface de la membrane peut, par elle-même, contribuer à une cholestase. Si le mécanisme principal par lequel l'absence de FIC1 exerce son effet sur le foie est l'induction d'une sensibilité de la membrane canalaire à des lésions par les sels biliaires, il est surprenant que le degré de perturbation morphologique observé en microscopie optique ne soit pas plus important. Ici encore pourtant, il semble probable que, même dans le foie, l'impact de la perte de la fonction de FIC1 soit réduit par la présence d'autres transporteurs de lipides et qu'une disparition totale de l'internalisation des aminophospholipides aboutirait probablement à une hépatopathie significativement plus sévère.

\section{Déficit en BSEP}

Le principal transporteur des acides biliaires de la membrane canalaire, appelé aujourd'hui BSEP, est codé par le gène $A B C B 11$. D’une façon similaire à celle observée dans le déficit en $\mathrm{FIC1}$, des patients présentant des phénotypes tant bénins (CIRB) [15] que sévères (CIFP) ont été identifiés $[16,17]$. La nomenclature est ici encore malheureuse, car il existe sans aucun doute des patients se répartissant sur la totalité du spectre entre ces deux extrêmes. Ce point est extrêmement important au plan clinique, car plusieurs patients dits CIRB présentent des caractéristiques suggérant une hépatopathie progressive et le fait qu'une maladie apparaisse tardivement et puisse sembler entrer en rémission ne doit pas être considéré comme une démonstration de l'absence de problèmes ultérieurs. Les mécanismes physiopathologiques aboutissant à un déficit en BSEP sont un peu plus faciles à comprendre. Une défaillance totale ou partielle du transport des acides biliaires, au niveau de la membrane canaliculaire, va exercer un effet manifeste sur la fonction hépatocellulaire. Il est clair que la perturbation des hépatocytes est beaucoup plus importante que celle observée dans le déficit en FIC1, avec souvent une transformation considérable en cellules géantes au cours de la petite enfance. La BSEP ne paraît pas être exprimée en dehors du foie et, de ce fait, les caractéristiques cliniques peuvent être totalement expliquées par une défaillance du transport des sels biliaires au niveau de la membrane canaliculaire. Les manifestations cliniques sont celles d'une cholestase sévère bien que, la bilirubine étant transportée de façon tout à fait distincte des sels biliaires, le degré d'ictère puisse fortement sous-estimer le degré de cholestase. Une transplantation hépatique est une excellente forme de traitement du déficit en BSEP.

Le carcinome hépatocellulaire ( $\mathrm{CHC}$ ) est extrêmement rare chez l'enfant, et le cholangiocarcinome l'est encore plus. D'autre part, l'hépatoblastome est l'affection maligne la plus fréquente affectant les hépatocytes, avec le CHC fibrolamellaire, qui est beaucoup plus rare. Ces deux affections malignes surviennent dans le contexte d'un foie normal, non dans celui d'une hépatopathie chronique. Chez l'enfant, un CHC s'observe lors de certaines maladies spécifiques, particulièrement la tyrosiné- 
mie classique et, à un moindre degré, les atteintes des stocks de glycogène. Dix jeunes enfants atteints de $\mathrm{CHC}$ et de déficit en BSEP ont été identifiés à la suite d'une recherche d'enfants atteints de CHC dans un contexte de tableau d'hépatite néonatale sévère [18]. Le risque exact est difficile à évaluer chez chaque patient, particulièrement parce que de nombreux patients sont transplantés avant l'apparition de cette complication. Dans une étude ultérieure, deux enfants atteints de cholangiocarcinomatose, se présentant ici encore dans le contexte d'une hépatite néonatale, se sont avérés atteints de déficit en BSEP [19]. Une analyse consécutive de 128 enfants atteints de déficit sévère en BSEP démontré par la présence de mutations a montré que 19 avaient été atteints de l'une ou de l'autre affection maligne [17]. Le risque paraît beaucoup plus important si le sujet présente deux mutations tronquant des protéines (38 contre 10\% dans d'autres génotypes). De nombreux patients avaient subi une transplantation précoce et aucune affection maligne n'était apparue. En l'absence de transplantation, le risque devrait être considérablement plus élevé. Tous ces patients étaient atteints d'une forme cliniquement sévère. Il n'existait aucune corrélation apparente entre la sévérité de la maladie et le risque d'affection maligne. Étant donné que d'autres enfants atteints de cholestase persistante ne semblent pas présenter d'affection maligne, on est amené à remettre en question le mécanisme de survenue de la modification néoplasique. Plusieurs des observations ci-dessus remettent en question le candidat apparemment évident que sont les lésions induites par les sels biliaires en rétention. Il est uniquement possible de supposer ce que pourrait être le mécanisme réel. Il est cependant certainement possible que la BSEP transporte normalement de faibles quantités de substances autres que des sels biliaires, ce qui entraînerait leur accumulation en son absence, ou, peut-être que la BSEP possède une autre fonction dont l'absence totale aboutirait à une affection maligne. Quel que soit le mécanisme, le risque est réel et doit être pris en compte lors de la détermination d'un mode de traitement quelconque, autre qu'une transplantation, qu'il soit pharmacologique, chirurgical ou génétique. Tous les traitements laisseront sans doute un risque d'affection maligne, nécessitant une surveillance très étroite.

\section{Déficit en protéine 3 de résistance multidrogue}

Au cours de l'évolution humaine, l'homme est devenu extrêmement efficient pour l'extraction des lipides de son alimentation. Dans la plupart des régions du monde où les calories abondent, nous en payons le prix. Cependant, pour atteindre une telle efficacité dans l'extraction des lipides de notre alimentation, nous avons développé la capacité à synthétiser des acides biliaires hautement détergents. Les acides biliaires, par leur nature même, sont toxiques. Quand ils sont retenus dans le foie, ils aboutissent à des atteintes sévères, comme observé dans le déficit en BSEP. Cependant, après leur transport dans la bile, ils atteignent des concentrations millimolaires. La bile ellemême peut donc induire des lésions sévères de l'arbre biliaire. De fait, quel que soit le facteur déclenchant une cholangite sclérosante ou une atrésie des voies biliaires, la bile sous forme d'acides biliaires est responsable de la plus grande partie des lésions. L'homme et de nombreux mammifères ont dû évoluer vers des mécanismes permettant de minimiser les effets toxiques des acides biliaires. Un mécanisme majeur de ce type consiste à permettre la formation de micelles mixtes. Elles se composent d'acides biliaires et d'autres lipides, particulièrement la phosphatidylcholine (PC). L'entrée de lipides, particulièrement de la PC, dans la bile afin de former des micelles est étroitement couplée au flux d'acides biliaires. Les données concernant le déficit en FIC1, discutées plus haut, suggèrent que les acides biliaires peuvent extraire des lipides de la membrane canaliculaire d'une façon plutôt non spécifique s'ils sont rendus disponibles. Normalement, ce phénomène ne se produit pas, car la plus grande partie de la membrane résiste aux détergents. Au contraire, la PC semble être extraite d'une façon hautement spécifique. La protéine 3 de résistance multidrogue (MDR3) est à présent reconnue comme essentielle pour l'entrée de la PC dans la bile [20]. La disponibilité de la PC dans le feuillet externe de la membrane canaliculaire paraît être étroitement contrôlée par MDR3. De ce fait, une perte même mineure de la fonction de MDR3 devrait modifier la composition de la bile. De fait, une gamme complète de phénotypes a été décrite en association à une réduction de la fonction de MDR3, dont une propension à la formation de calculs biliaires $[21,22]$. Des patients chez lesquels une seule copie est défectueuse peuvent présenter des symptômes dans certaines circonstances, par exemple au cours d'une grossesse $[23,24]$, bien que ne présentant pas d'autres troubles. À l'extrémité sévère du spectre, la CIFP semble relativement rare. Cette affection est facilement distinguée des déficits en FIC1 et BSEP car elle est associée à des taux élevés de $\gamma$-glutamyl transférase circulante. Cependant, des mutations de MDR3 étant de plus en plus recherchées, une maladie liée à MDR3 d'apparition tardive, dont une cholangite sclérosante des petits canaux, une cirrhose biliaire primitive AMA-négative et 
même une cirrhose cryptogénique ont été identifiées. Il demeure à déterminer si MDR3 joue réellement un rôle majeur dans la cholélithiase cholestérolique. Des mutations de ce gène ne sont cependant pas rares dans des arbres généalogiques où de nombreux sujets sont atteints, particulièrement si les calculs sont intra-hépatiques [25].

\section{Conclusions}

Ces dix dernières années, les connaissances des mécanismes moléculaires intervenant dans la formation de la bile et la cholestase se sont spectaculairement améliorées. Une analyse de familles distinguant différentes formes d'hépatopathie a joué un rôle significatif dans ces progrès. De plus, nous pouvons maintenant constater que certaines affections à début tardif ont, dans certains cas, une cause génétique et, dans d'autres, une prédisposition génétique. Le degré auquel les atteintes hépatiques à début tardif sont réellement des versions moins sévères de ces affections demeure à déterminer. Nous n'avons probablement fait que gratter la surface à ce jour. En pédiatrie, il est maintenant essentiel de convertir ces connaissances en évolutions plus favorables pour les patients. Aux ÉtatsUnis, il s'agit précisément de l'objectif d'un groupe coopératif multicentrique [26]. Une grande partie des données rétrospectives publiées a été recueillie chez des patients que nous considérerions aujourd'hui comme mal caractérisés. Avec la nette amélioration de nos techniques et connaissances, nous devrions désormais être capables de poser le diagnostic plus rapidement, de mieux comprendre les processus pathologiques et de planifier un traitement et des essais à la lumière de ces connaissances.

\section{Bibliographie}

1 Biliary Atresia Research Consortium. http:// www.barcnetwork.org/.

2 European Federation of Biliary Atresia Research. http://www.orpha.net/nestasso/ EFBAR/.

3 European Biliary Atresia Registry. http:// www.biliary-atresia.com/index_frameset ebar.html.

-4 Liu C, Aronow BJ, Jegga AG, et al: Novel resequencing chip customized to diagnose mutations in patients with inherited syndromes of intrahepatic cholestasis. Gastroenterology 2007;132:119-126.

5 Oda T, Elkahloun AG, Pike BL, et al: Mutations in the human Jagged1 gene are responsible for Alagille syndrome. Nat Genet 1997; 16:235-242.

6 Li L, Krantz ID, Deng Y, et al: Alagille syndrome is caused by mutations in human Jagged1, which encodes a ligand for Notch1. Nat Genet 1997;16:243-251.

-7 McDaniell R, Warthen DM, Sanchez-Lara $\mathrm{PA}$, et al: NOTCH2 mutations cause Alagille syndrome, a heterogeneous disorder of the notch signaling pathway. Am J Hum Genet 2006;79:169-173.

8 Xue Y, Gao X, Lindsell CE, et al: Embryonic lethality and vascular defects in mice lacking the Notch ligand Jagged1. Hum Mol Genet 1999;8:723-730.

-9 Warthen DM, Moore EC, Kamath BM, et al: Jagged1 (JAG1) mutations in Alagille syndrome: increasing the mutation detection rate. Hum Mutat 2006;27:436-443.

10 Gissen P, Johnson CA, Morgan NV, et al: Mutations in VPS33B, encoding a regulator of SNARE-dependent membrane fusion, cause arthrogryposis-renal dysfunction- cholestasis (ARC) syndrome. Nat Genet 2004;36:400-404.

11 Bull LN, Mahmoodi V, Baker AJ, et al: VPS33B mutation with ichthyosis, cholestasis, and renal dysfunction but without arthrogryposis: incomplete ARC syndrome phenotype. J Pediatr 2006;148:269-271.

12 Bull LN, van Eijk MJ, Pawlikowska L, et al: A gene encoding a P-type ATPase mutated in two forms of hereditary cholestasis. Nat Genet 1998;18:219-224.

13 van Mil SW, Klomp LW, Bull LN, et al: FIC1 disease: a spectrum of intrahepatic cholestatic disorders. Semin Liver Dis 2001;21:535544.

14 Paulusma CC, Groen A, Kunne C, et al: Atp8b1 deficiency in mice reduces resistance of the canalicular membrane to hydrophobic bile salts and impairs bile salt transport. Hepatology 2006;44:195-204.

-15 van Mil SW, van der Woerd WL, van der Brugge $G$, et al: Benign recurrent intrahepatic cholestasis type 2 is caused by mutations in ABCB11. Gastroenterology 2004;127:379384.

16 Strautnieks SS, Bull LN, Knisely AS, et al: A gene encoding a liver-specific ABC transporter is mutated in progressive familial intrahepatic cholestasis. Nat Genet 1998;20: 233-238.

17 Strautnieks SS, Byrne JA, Pawlikowska L, et al: Severe bile salt export pump deficiency: 82 different ABCB11 mutations in 109 families. Gastroenterology 2008;134:1203-1214.

18 Knisely AS, Strautnieks SS, Meier Y, et al: Hepatocellular carcinoma in ten children under five years old with bile salt export pump deficiency. Hepatology 2006;44:478-486.
19 Scheimann AO, Strautnieks SS, Knisely AS, et al: Mutations in bile salt export pump (ABCB11) in two children with progressive familial intrahepatic cholestasis and cholangiocarcinoma. J Pediatr 2007;150:556-559.

20 de Vree JM, Jacquemin E, Sturm E, et al: Mutations in the MDR3 gene cause progressive familial intrahepatic cholestasis. Proc Natl Acad Sci USA 1998;95:282-287.

21 Rosmorduc O, Hermelin B, Poupon R: MDR3 gene defect in adults with symptomatic intrahepatic and gallbladder cholesterol cholelithiasis. Gastroenterology 2001;120: 1459-1467.

-22 Jacquemin E, De Vree JM, Cresteil D, et al: The wide spectrum of multidrug resistance 3 deficiency: from neonatal cholestasis to cirrhosis of adulthood. Gastroenterology 2001;120:1448-1458.

23 Dixon PH, Weerasekera N, Linton KJ, et al: Heterozygous MDR3 missense mutation associated with intrahepatic cholestasis of pregnancy: evidence for a defect in protein trafficking. Hum Mol Genet 2000;9:12091217.

24 Jacquemin E, Cresteil D, Manouvrier S, et al: Heterozygous non-sense mutation of the MDR3 gene in familial intrahepatic cholestasis of pregnancy. Lancet 1999;353:210211.

25 Rosmorduc O, Poupon R: Low phospholipid associated cholelithiasis: association with mutation in the MDR3/ABCB4 gene. Orphanet J Rare Dis 2007;2:29.

26 Cholestatic Liver Disease Consortium. http://rarediseasesnetwork.epi.usf.edu/clic/ index.htm. 